\title{
APORTES DESDE LAS CIENCIAS BIOLÓGICAS A LA TEORÍA DE LA GESTIÓN*
}

\author{
PAULA VIVIANA ROBAYO ACUÑA**, ALEXANDRA MONTOYA RESTREPO*** \\ \& OSCAR CASTELLANOS DOMÍNGUEZ**:** \\ UNIVERSIDAD NACIONAL DE COLOMBIA
}

Recibido/ Received/ Recebido: 14/12/2010 - Aceptado/ Accepted / Aprovado: 10/05/2011

\begin{abstract}
Resumen
Las metáforas biológicas han sido aplicadas en los modelos de gestión organizacional para fortalecer su conceptualización y entendimiento, sin embargo se requiere hacer un seguimiento bibliométrico de estos aportes con el fin de articular dicha información. El objetivo del presente documento es destacar los resultados obtenidos para establecer el estado del arte, enfoques y aportes del desarrollo de la aplicación de los modelos biológicos en las ciencias administrativas. De los resultados obtenidos se encontró que existe un fuerte posicionamiento de la aplicación de los conceptos biológicos en la implementación de procesos productivos, como una forma de administrar eficientemente la producción. Adicionalmente, la generación de la comparación está relacionada con las formas de organización del medio ambiente para ser aplicadas a las estructuras organizacionales. No obstante, el análisis realizado muestra que la dinámica de publicaciones sobre el aporte de las biociencias a la gestión tiene mucho potencial y ha sido diverso conceptualmente.
\end{abstract}

Palabras clave: Metáfora organizacional, metáfora biológica, modelos de gestión organizacional.

\section{CONTRIBUTIONS FROM LIFE SCIENCES TO MANAGEMENT THEORY}

\begin{abstract}
Biological metaphors have been applied to organizational management models, however, a bibliometrical monitoring of these contributions needs to be done with the purpose of articulating such information. The objective of this paper is to outline the results to establish the state of art, approaches and contributions of biological models application development in administrative sciences. From the obtained results, it was found that there is a strong positioning of biological concepts application in the implementation of productive processes as a form to manage production efficiently. Additionally, the generation of comparing is related to organization forms of the environment to be applied in organizational structures. However, the analysis made shows that
\end{abstract}

\footnotetext{
El presente trabajo hace parte de la línea de investigación BioGestión y pertenece al proyecto Fortalecimiento conceptual del Grupo de Investigación y Desarrollo, en gestión, productividad y competitividad: Identificación de tendencias sobre el aporte de las biociencias a la Gestión. Bogotá, Universidad Nacional de Colombia. Los autores manifiestan su agradecimiento a la ingeniera Sandra Lorena Fonseca, por su trabajo dentro del proyecto.

* Administradora de Empresas (2007) de la Universidad Nacional de Colombia, sede Bogotá. Estudios de Maestría en Administración de la Universidad Nacional de Colombia, sede Bogotá. Correo electrónico: purobayoa@unal.edu.co.

*** Administradora de Empresas, Magíster en Administración, Ph.D en Ciencias Económicas, Profesora Asociada, Universidad Nacional de Colombia, sede Bogotá. Integrante del Grupo de Investigación y Desarrollo, en Gestión, Productividad y Competitividad, BIOGESTIÓN. Correo electrónico: lamontoyar@unal.edu.co

Ingeniero Químico (1990), Magíster en Ciencias Técnicas (1992), Magíster en Administración (2002), Ph.D. en Química (1995), Estudios posdoctorales en Biotecnología de enzimas (2005) Profesor Asociado, Universidad Nacional de Colombia, Director del Grupo de Investigación y Desarrollo, en Gestión, Productividad y Competitividad, BIOGESTIÓN. Correo electrónico: ofcastellanosd@unal.edu.co
} 
publication dynamic on biosciences contribution to management has a lot potential and has been conceptually diverse.

Keywords: Organizational metaphor, biological metaphor, organizational management models.

\title{
CONTRIBUIÇÕES DAS CIÊNCIAS BIOLÓGICAS À TEORIA DA ADMINISTRAÇÃO
}

\begin{abstract}
Resumo
Para melhorar a conceituação e a compreensão dos modelos de gestão organizacional, têm-se utilizado metáforas biológicas nos modelos de gestão organizacional. No entanto, é necessário fazer um seguimento bibliométrico dessas contribuições para articular essa informação. O objetivo deste trabalho é destacar os resultados para estabelecer o estado da arte, as abordagens e as contribuições do desenvolvimento da aplicação dos modelos biológicos na ciência da administração. Os resultados mostraram um forte posicionamento na aplicação de conceitos biológicos no estabelecimento de processos de produção como uma forma de gerenciar com eficiência a produção. Além disso, a comparação está relacionada às formas de organização do ambiente para aplicá-las às estruturas organizacionais. Não obstante, esta análise mostra que a dinâmica de publicações sobre a contribuição das ciências biológicas para a gestão tem um grande potencial e conceitualmente é diferente.

Palavras chave: Metáfora organizacional, metáfora biológica, modelos de gestão organizacional.
\end{abstract}

Acuña, P., Montoya, A. \& Castellanos, O. (2011) Aportes desde las ciencias biológicas a la teoría de la gestión. En: Revista de la Facultad de Ciencias Económicas de la Universidad Militar Nueva Granada. rev.fac.cienc.econ, XIX (1).

JEL: M19.

\section{Introducción}

El estudio de las organizaciones como centro de la investigación de la teoría administrativa se nutre de conceptos, instrumentos e incluso de ciencias afines para su desarrollo. Es así como puede observarse que la administración se fundamenta en ciencias como la economía, la ingeniería, la psicología, la sociología, entre otras (Montoya, 2010).

La contribución de otros campos ha sido evidente desde los inicios de las teorías administrativas, es el caso de la visión mecanicista (el hombre y la organización como máquina), que caracterizó la Administración Científica de Taylor y que se sustentó en la ingeniería. En un segundo caso, aparece la contribución de psicólogos, sociólogos y de las ciencias de la conducta con los aportes de Mayo a las teorías motivacionales, así como las teorías de desarrollo de la publicidad (Morgan, 2006). De otra parte, para facilitar su entendimiento la administración también se provee de nuevas formas de comunicación y de instrumentos alternativos de análisis que le permiten aprender mejor, en función de la comprensión, la complejidad de las organizaciones y su gestión; este es el caso de metáforas, por medio de las cuales se ha inducido la comparación de particularidades de su objeto de estudio, lo que se acompaña con elementos y objetos conocidos por otras áreas del saber. De esta manera se facilitan el estudio y la comprensión de las organizaciones e incluso se tratan de deducir aspectos importantes de ellas, los que así podrán ser asimilados más fácilmente (Castellanos, 2004).

1 Las metáforas son provenientes del griego meta, "más allá», y forein, "pasar», "llevar» y son definidas como un recurso literario que consiste en identificar dos términos entre los cuales existe alguna semejanza. Uno de los términos es el literal y el otro el que se 
Las metáforas ${ }^{1}$ son una parte integrante del lenguaje y del pensamiento y aparecen en las conversaciones cotidianas, aún sin que en ellas sean tenidas conscientemente en cuenta. Se utilizan, a menudo, cuando se quiere comunicar un concepto abstracto de una manera familiar y accesible, es por ello que Lores (2006) afirma, que una parte del lenguaje está basado en las metáforas.

En términos epistemológicos la metáfora proporciona nuevos conocimientos al crear una imagen; relaciona dialécticamente lo abstracto con lo concreto; la lógica formal con la lógica Ilustracióntiva; la sintáctica y la semántica con la pragmática; y también la complejidad con la simplicidad (Mendoza, 2005) Ahora, si bien se encuentran diversas metáforas desde diferentes disciplinas aplicadas a la Gestión, este artículo presenta una revisión específicamente desde las ciencias biológicas, ya que es justamente la biología, como ciencia natural no ceñida a leyes determinísticas e inmutables como la física y la química, representadas frecuentemente por formulaciones matemáticas, que presenta suficiente flexibilidad en sus fundamentos y su interpretación y que se ha convertido en la ciencia en la cual los diferentes autores han encontrado el ámbito referencial para retomar conceptos y modelos, aplicables al desarrollo económico y empresarial, teniendo en cuenta que los actuales ambientes cambiantes exigen el aprendizaje dinámico en la empresa, en la cual la ciencia biológica ha elaborado una externalidad conceptual positiva, a través de un conjunto de esquemas de pensamiento especialmente útiles para el estudio de sistemas dinámicos (Montoya, 2010).

Las biociencias han aportado a la gestión organizacional -a través de distintas metáforas- un mayor entendimiento de la organización y su manejo gerencial, en este sentido se pueden mencionar las siguientes:
Tabla 1. Principales autores que han trabajado la metáfora biológica

\begin{tabular}{|c|c|}
\hline Autor & Metáfora \\
\hline Bateson, 1972 & Pensamiento sistémico \\
\hline Beer, 1977 & Relación con otras ciencias \\
\hline Brigs-Peat, 1989 & Organización como cerebro \\
\hline Capra, 1998 & Como organismo vivo \\
\hline $\begin{array}{l}\text { Fischer-Kowalski, } \\
2007\end{array}$ & Metabolismo socioeconómico \\
\hline Gell-Mann, 1999 & Sistemas complejos adaptativos \\
\hline $\begin{array}{l}\text { Gouillart-Kelly, } \\
2007\end{array}$ & Bioingeniería \\
\hline $\begin{array}{l}\text { Hannan \& Carroll, } \\
1992\end{array}$ & Concepto de nicho organizacional \\
\hline $\begin{array}{l}\text { Hannan-Freeman, } \\
1977,2003\end{array}$ & $\begin{array}{l}\text { Ecosistemas organizacionales y } \\
\text { nichos }\end{array}$ \\
\hline $\begin{array}{l}\text { Hatch, 1997; } \\
\text { Becker, } 2007\end{array}$ & Ecología de organizaciones \\
\hline Holland, 2003 & Sistemas complejos adaptables \\
\hline Illera, 1982 & Estructura humana \\
\hline Kauffman, 2003 & $\begin{array}{l}\text { Agentes autónomos, relieves adapta- } \\
\text { tivos }\end{array}$ \\
\hline $\begin{array}{l}\text { Lara \& Taboada, } \\
2005\end{array}$ & $\begin{array}{l}\text { Cooperación empresarial como siste- } \\
\text { ma biológico. } \\
\text { Modelos coevolutivos organizacionales }\end{array}$ \\
\hline Lessem, 1990 & Arboles de conocimiento \\
\hline Lima, 2001 & Modelo evolucionista de gestión \\
\hline Martínez, 1986 & Bioeconomía \\
\hline $\begin{array}{l}\text { Maturana-Varela, } \\
1990\end{array}$ & Como proceso cognitivo \\
\hline Montoya,1999 & Como sistema genético \\
\hline Moore, 1996, 2005 & Ecosistema de negocios \\
\hline Morgan,1991 & Cerebro \\
\hline Morin,1995 & $\begin{array}{l}\text { Organizaciones como sistemas com- } \\
\text { plejos }\end{array}$ \\
\hline
\end{tabular}

usa en sentido Ilustracióndo. Este recurso puede ser estudiado por su doble componente: el primero, porque puede sustituir una enunciación con otra prestada de otro campo; y el segundo, que se refiere a la transgresión que la metáfora ejerce sobre la estructura significativa del lenguaje (Mendoza, 2005) En particular se resalta aquí el primer componente porque es muy útil dentro del estudio de las organizaciones, ya que permite utilizar el lenguaje de ciencias hermanas para ampliar y mejorar el entendimiento de los conceptos administrativos.

2 Fuente: Montoya, Montoya \& Castellanos (2009). 


\begin{tabular}{|l|l|}
\hline $\begin{array}{l}\text { Nalebuff \& Bran- } \\
\text { derburger, 1996 } \\
\text { Shapiro \& Varian, } \\
1998 .\end{array}$ & Coopetencia \\
\hline Nooteboom, 2003 & $\begin{array}{l}\text { Modelos de integración con enfoque } \\
\text { evolucionista }\end{array}$ \\
\hline Pauli, 1997 & Como sistema inmunológico \\
\hline $\begin{array}{l}\text { Pech \& Oakley, } \\
2005\end{array}$ & Hormesis organizacional \\
\hline Prigogine, 1967 & Como red autopoyética \\
\hline Rammel, 2007 & Co- Management \\
\hline Sheldrake, 1990 & Campos morfo genéticos, colonias. \\
\hline Tinaur, 1999 & Parasitismo organizacional \\
\hline Wheatley, 1996 & Auto organización \\
\hline $\begin{array}{l}\text { Zimmer, 2007; } \\
\text { Pereira, 2008 }\end{array}$ & Swarm Intelligence \\
\hline
\end{tabular}

Entre los aportes en Latinoamérica, se encuentra la metáfora de la Gerencia Genética planteada por Montoya (1999), en la cual se estructura la propuesta de un modelo que busca relacionar la naturaleza de las funciones de transmisión, codificación y almacenamiento de la información genética con los procesos de gestión y dirección en las organizaciones. Es posible hacer la metáfora porque el sistema genético, que maneja la información de un organismo, la define como un signo o conjunto de signos que impulsan a la acción, lo cual a su vez puede ser base de un modelo para la toma de decisiones en condiciones de incertidumbre en una organización y el concepto de Biogestión que surge del reconocimiento de la interacción explícita entre la gestión y la biología.

A partir de esta premisa, la biogestión se propone integrar en un contexto holístico extendido diferentes aspectos de las teorías administrativas con los conceptos y los sistemas de las ciencias biológicas. Esta nueva forma conceptual de integración interdisciplinaria tiene un carácter transversal que le permite interactuar o servir como instrumento metodológico y articulador coherente de desarrollos actuales, tales como: el biocomercio, la bioprospectiva, la bioética y la bioseguridad, entendidos en contextos estructurados. La Biogestión se plantea como un paso en la cadena que busca a partir de las necesidades individuales y sociales, mediante el uso de la tecnología, aplicar los conceptos de lo biológico de forma interdisciplinar para generar bienes $y$ servicios de calidad (Castellanos, 2002).

\section{Metodología}

A partir de los antecedentes metafóricos descritos anteriormente y lo interesante que este proceso resulta para la creación de nuevas aplicaciones que permitan tener un entendimiento mayor de la complejidad del estudio de las organizaciones y conocer las tendencias recientes del aporte de las ciencias biológicas al desarrollo de la empresa y su gestión, se presentan a continuación la metodología usada y los resultados obtenidos en un proyecto de investigación ${ }^{3}$ que con anterioridad se desarrolló con el propósito de realizar una búsqueda de referentes internacionales para establecer el estado del arte, las tendencias y las temáticas del desarrollo de la aplicación de nuevos conceptos o modelos biológicos en las ciencias administrativas, con énfasis en los modelos de integración para que las empresas puedan mejorar su competitividad y amplíen su supervivencia dentro del entorno cambiante y globalizado, y así fortalecer ésta base conceptual y la utilización de la metáfora biológica para llegar a las aplicaciones que actualmente en gestión se han realizado sobre la misma, teniendo en cuenta que este enfoque no ha sido ampliamente difundido.

En la Ilustración 1 se presenta la metodología que se para la investigación y sobre la cual se obtuvieron los resultados que se presentan posteriormente. Inicialmente para el desarrollo de esta vigilancia tecnológica se seleccionaron cuatro bases de datos del conjunto disponible en el Sistema de Bibliotecas de la Universidad Nacional de Colombia ${ }^{4}$, entre

\footnotetext{
Montoya, Robayo, Castellanos \& Fonseca, (2008).

4 El sistema de Bibliotecas SINAB, se articula a través del portal http://www.sinab.unal.edu.co, integra los recursos bibliográficos para ofrecer información académica y científica (Bases de datos, catálogos, libros y revistas electrónicas, entre otros).
} 
ellas: Emerald, Isi Web Knowledge, Science Direct y Blackwell Synergy. Los criterios considerados para este procedimiento incluyeron su pertinencia con la temática, la cobertura, las opciones de búsqueda, facilidad en el manejo y descarga de datos, posteriormente se plantearon las ecuaciones de búsqueda que tuvieran las palabras claves en donde se relacionaba el concepto biológico con el de gestión, a partir de ello, se descargaron los artículos que tenían mayor pertinencia y se clasificó la información. Con ella se obtuvieron dos tipos de indicadores, los relacionales y los de actividad.

Para la definición de las ecuaciones de búsqueda se establecieron tres categorías de palabras enmarcadas y relacionadas con los conceptos de gestión, biología e integración. Estas categorías son: conceptos biológicos, atributos de la vida y conceptos relacionados con los sistemas de integración. Posteriormente se construyeron las ecuaciones de búsqueda, proceso en el cual se tuvieron en cuenta aspectos relevantes de cada una de las categorías mencionadas y su correspondiente denominación en inglés. Para obtener el conjunto de registros adecuado fue necesario recurrir a diferentes ecuaciones, analizar y depurar los registros obtenidos y en algunos casos reformular la búsqueda. El estudio se realizó para un lapso de tiempo de 10 años (Enero 1998 - Noviembre 2008), utilizando los campos de búsqueda de título, palabras clave y en algunas ocasiones, dependiendo de la base de datos el resumen. En la Tabla 2 se presentan las ecuaciones de búsqueda utilizadas.

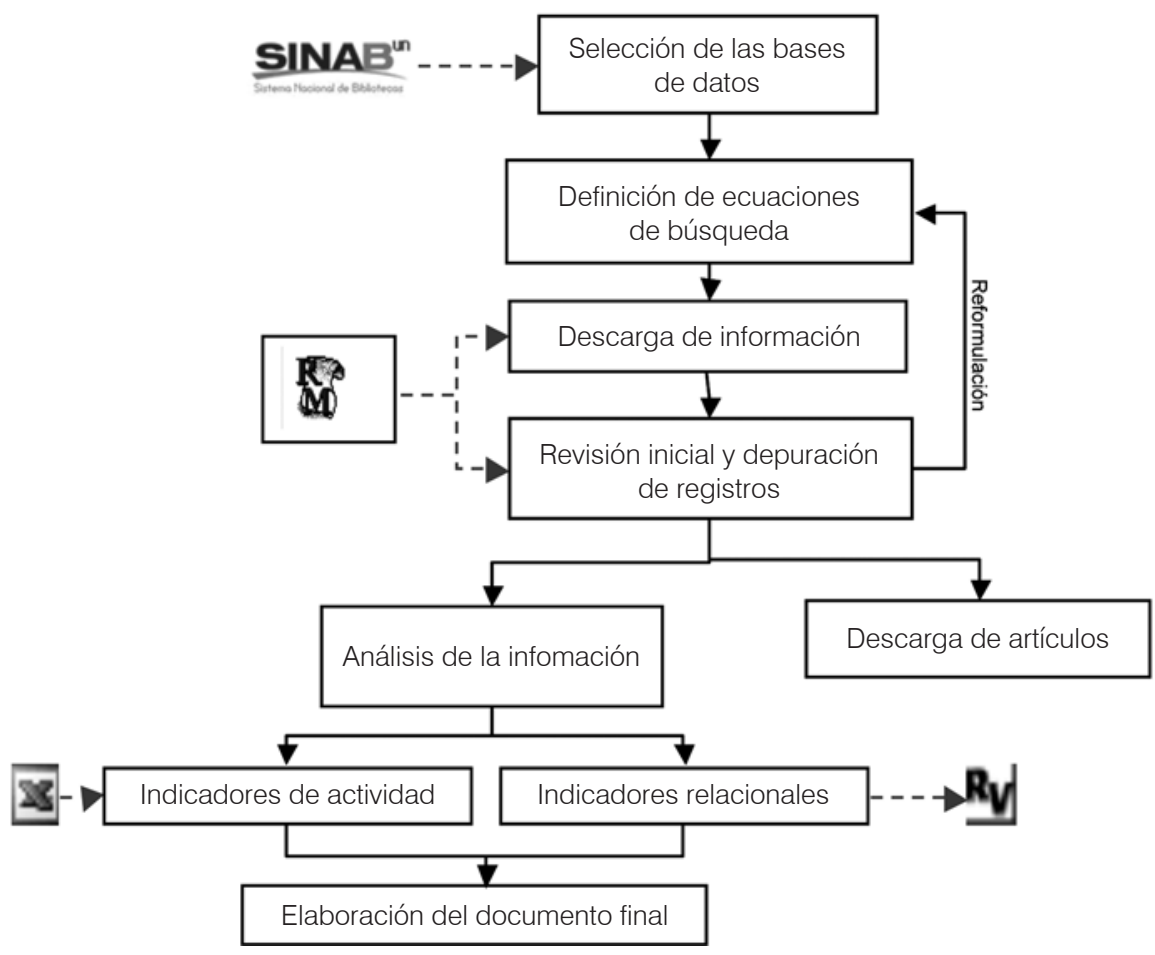

Ilustración 1. Metodología para la identificación de tendencias a nivel Internacional ${ }^{5}$

\footnotetext{
5 Elaboración propia.
} 
Tabla 2. Ecuaciones de búsqueda ${ }^{6}$

\begin{tabular}{|c|c|c|}
\hline EJE & TEMA & ECUACIÓN DE BÚSQUEDA \\
\hline \multirow{6}{*}{$\begin{array}{l}\text { Conceptos } \\
\text { Biológicos }\end{array}$} & ANALOGÍA & (analogy AND manag*) \\
\hline & BIOLÓGICO & $($ biolog* AND manag*) \\
\hline & CICLO DE VIDA & (life cycle AND manag*) \\
\hline & GENÉTICA & $($ genetic AND manag*) \\
\hline & METÁFORA BIOLÓGICA & ("biolog* metaphor" AND manag*) \\
\hline & ORGANISMO & ('organism AND manag*) \\
\hline \multirow{5}{*}{ Atributos de la vida } & ORGANIZACIÓN ORGÁNICA & ("organic organization" AND manag*) \\
\hline & ADAPTATIVO & $\left(\right.$ adap* AND manag*) $^{*}$ \\
\hline & EVOLUTIVO & $($ evolut* AND manag*) \\
\hline & ORGÁNICO & (organic AND manag*) \\
\hline & ORGANIZACIÓN VIVIENTE & ("living organization" AND manag*) \\
\hline \multirow{12}{*}{$\begin{array}{l}\text { Conceptos de } \\
\text { Integración }\end{array}$} & SOCIOBIOLOGÍA & $\left(\right.$ sociobiolog ${ }^{\star}$ AND manag*) \\
\hline & ECOSISTEMA & $\left(\right.$ ecosystem and manag $\left.{ }^{\star}\right)$ \\
\hline & ECOLOGÍA & $($ ecology* AND manag*) OR (organizational ecolog*) \\
\hline & MORTALIDAD & (mortality AND manag*) OR (mortality AND organizat*) \\
\hline & $\mathrm{NICHO}$ & (niche AND manag*) OR (niche AND organizat*) \\
\hline & SINERGÍA & (synerg* AND manag*) OR (synerg* AND organizat*) \\
\hline & INERCIA & (inertia AND manag*) OR (inertia AND organizat*) \\
\hline & COOPERACIÓN & $\begin{array}{l}\text { (cooperation AND manag*) OR (cooperation AND organizat*) OR (coope- } \\
\text { ration AND integration) }\end{array}$ \\
\hline & CLUSTER & $\begin{array}{l}\text { (cluster AND manag*) OR (cluster AND organizat*) OR (cluster AND inte- } \\
\text { gration) }\end{array}$ \\
\hline & CONGLOMERADO & $\begin{array}{l}\text { (conglomerate AND manag*) OR (conglometate AND organizat**) OR (con- } \\
\text { glomerate AND integration) }\end{array}$ \\
\hline & AGLOMERACIÓN & $\begin{array}{l}\left.\text { (agglometation AND manag }{ }^{\star}\right) \\
\text { (aglomeration AND integration) }\end{array}$ \\
\hline & RED & $\begin{array}{l}\text { (network AND manag*) OR (network AND organizat*) OR (network AND in- } \\
\text { tegration) }\end{array}$ \\
\hline
\end{tabular}

\section{Análisis y Resultados}

A partir de los programas Excel(C) y Refviz $($ se analizaron cada uno de los conjuntos de datos determinados por las temáticas anteriormente mencionadas, teniendo en cuenta, que el número de registros encontrados no fue muy amplio. En este paso se establecieron por cada uno de los ejes temáticos los siguientes indicadores de actividad: número de publicaciones por año, autores con mayor número de publicaciones, revistas con mayor número de publicaciones y palabras claves con mayor índice de frecuencia, a partir de los cuales se realizó el correspondiente análisis. Por último, se desarrollaron los mapas tecnológicos que presentan las relaciones encontradas entre cada una de las palabras aportadas por los artículos seleccionados.

6 Elaboración propia. 


\section{Conceptos Biológicos}

En este eje se utilizaron seis palabras para realizar la búsqueda, para un total de 40 registros netos, discriminados tal como se muestra en la Tabla 3. Es importante anotar que en este eje no se encontraron para el año 2000 artículos relacionados con las palabras seleccionadas. No hay una razón evidente que justifique este comportamiento.

Tabla 3. Registros encontrados relacionados con conceptos biológicos ${ }^{7}$

\begin{tabular}{|c|c|c|}
\hline Eje & Tema & Cantidad de registros \\
\hline \multirow{6}{*}{$\begin{array}{l}\mathscr{0} \\
0 \\
.0 \\
\frac{0}{0} \\
\frac{0}{0} \\
00 \\
0 \\
0 \\
0 \\
\frac{0}{0} \\
0 \\
0 \\
0 \\
0\end{array}$} & ANALOGÍA & 8 \\
\hline & BIOLÓGICO & 4 \\
\hline & CICLO DE VIDA & 19 \\
\hline & GENÉTICA & 5 \\
\hline & METÁFORA BIOLÓGICA & 4 \\
\hline & ORGANISMO & 0 \\
\hline \multicolumn{2}{|c|}{ Registros encontrados } & 40 \\
\hline \multicolumn{2}{|c|}{ Depurados } & 0 \\
\hline \multicolumn{2}{|c|}{ Total registros } & 40 \\
\hline
\end{tabular}

En la llustración 2, se visualiza que desde 1998 hasta el año 2003 existió un crecimiento moderado en el número de publicaciones, aunque es de notar que la publicación es escasa frente a otros temas que reportan un mayor número de escritos. Se evidenciaron dos picos de publicación, uno en el año 2003, seguido del año 2005, con 8 y 9 publicaciones respectivamente, esto puede estar dado por un boom generado por el tema de ciclo de vida y algoritmos genéticos aplicados, lo que llamó la atención de los autores a investigar y escribir sobre estos temas en estos dos años. No existieron coincidencias en los journals, lo que quiere decir que no hay una publicación o edición especial del tema.En relación con el índice de autores con mayor número de publicaciones, este análisis arrojó un total de 89 autores cada uno con una publicación, resaltando así la gran diversidad del tema, la falta de continuidad de los autores en el mismo y una tendencia naciente por este tipo de temáticas.

Estos autores se concentraron en 40 instituciones diferentes, y se reunieron en 12 países, los de mayor preeminencia fueron: Estados Unidos, Inglaterra y Sur Korea cada uno con una participación de $30,3 \%, 12,4 \%$ y $6,7 \%$ respectivamente, abarcando el $50 \%$ de la contribución total.

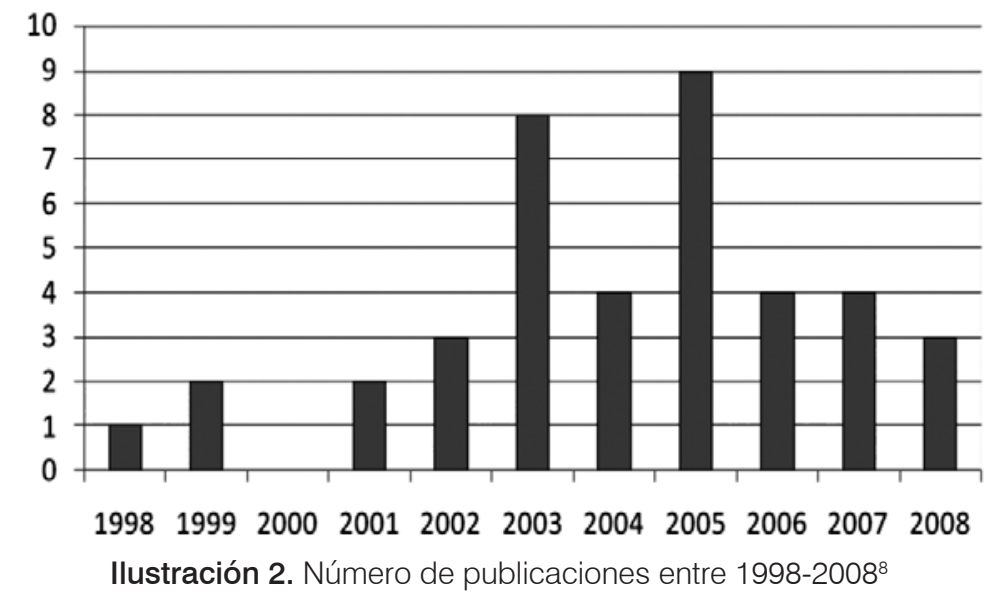

Elaboración propia.

8 Elaboración propia. 
Estados Unidos presentó trabajos con una tendencia hacia temas relacionados con las disciplinas de la gestión, la ingeniería de producción y la ingeniería industrial. De otra parte, Inglaterra desarrolló sus trabajos en el área temática de la gestión y los negocios. Y Sur Korea, no tuvo una disciplina representativa, sin embargo, se inclinó por temáticas de tecnología como la Inteligencia Artificial, la Ciencia de la Computación, la Gestión de la Producción de Tecnología y la Ingeniería Eléctrica y Electrónica.

Analizando las palabras claves con mayor frecuencia de aparición en cada uno de los artículos y años estudiados, los términos encontrados hicieron referencia a modelos y diseños utilizados en las diferentes áreas, entre ellas los sistemas y la ingeniería con aplicación de conceptos biológicos, en este caso la mayoría centrados en algoritmos genéticos, analogías y el ciclo de vida. Los primeros siete términos (management, designs, research, science, model, lifecycle, products) aparecen en promedio durante siete años de los 10 estudiados, adicionalmente estas palabras claves aparecen siempre en el 2003, 2005, 2006, 2007 y 2008, lo que permite decir que existe una continuidad en el tiempo y un naciente interés por estas temáticas. Para este análisis se tuvieron en cuenta aquellos conceptos que obtuvieron un número igual o mayor a tres apariciones únicamente.

Los primeros siete términos se encuentran relacionados entre sí, y se pueden articular alrededor de dos temáticas principalmente:

- La teoría de ciclo de vida del producto: Hace alusión a investigaciones en las que se buscan soluciones y modelos holísticos para el diseño $y$ desarrollo de nuevos productos, en los cuales se minimice el costo y se generen planes con una vista integral que les permita ser confiables, de calidad y se encuentre siempre disponibles.

- La aplicación de algoritmos genéticos en modelos de producción y financieros: Nacen como formas y cálculos matemáticos que permiten resolver problemas referentes a los procesos de producción y financieros relacionados con los portafolios de inversión. Los algoritmos genéticos son una técnica de optimización estocástica aplicada por Holland en 1975 en la Universidad de Michigan, y se generaron como una aplicación de la biología para guiar la búsqueda de una solución óptima en los diferentes portafolios de acciones que son posibles de armar. Sin embargo, los artículos hacen referencia a estudios de las tendencias y aplicaciones que se le han dado, mostrando las diferentes áreas potenciales de investigación adicionales a las de solución de inconvenientes en la gestión de producción y operaciones.

Ecological Economics, International Journal of Production Research, Academy Management of Executive, Cirp Annals- Manufacturing Technology y Journal Operations Management hicieron parte de las cinco revistas con mayor número de publicaciones de un total de 33 en donde se encontraron escritos del tema. Sin embargo, el número máximo de publicaciones por cada una de ellas fue de 3 artículos, realizados en diferentes años del período estudiado. Las cinco primeras revistas, hacen énfasis en tópicos con tendencias en manufactura, industria y operaciones. En este sentido, la revista Ecological economics presenta temáticas generales de ciencias sociales, sectores industriales, y la problemática medioambiental y económica; en Academy of management executive los artículos proporcionaron información y herramientas basadas en los recientes adelantos de la teoría de gestión e investigación. International journal of production research y el Journal of operations management hacen énfasis en temáticas de tecnología, manufacturas, administración de la producción y de operaciones. La revista CIRP Annals - Manufacturing Technology, resalta la tecnología, la maquinaria e ingeniería mecánica, adicionalmente, los sistemas de producción y los sistemas organizacionales.

Se presenta el agrupamiento de los documentos organizados por contenidos temáticos resaltando los temas con mayor relevancia, para este caso existen 6 grupos, de los cuales 3 concentran la mayor cantidad de referencias. De esta forma el clúster No. 5 relaciona catorce referencias relaciona los términos Management, life y cycle. Los artículos que le 
componen se centraron en las operaciones de producción, y en el ciclo de vida de los productos analizados desde diversas perspectivas, por ejemplo, la adaptación de la cadena de suministro como una competencia efectiva que apoya la gestión del ciclo de vida de los productos (Juttner, Godsell, Christopher, 2006). De otra parte, Liao \& Stonebraker (2006), realizan un estudio y presentan un modelo en el que sostienen que la fase de ciclo de vida está asociado con variables de las diversas dimensiones de la integración de la cadena de suministro, y que la complejidad y la munificencia del medio ambiente tienen un peso significativo en la moderación de los efectos sobre las relaciones.

Respecto a las palabras de complejidad y cambio, en los artículos se presenta la Teoría de la complejidad, como un término genérico para una serie de teorías, ideas y programas de investigación que se derivan de las disciplinas científicas tales como la meteorología, la biología, física, química y matemáticas, como una forma de entender y de cambiar las organizaciones (Burnes, 2005).

El cluster 1 con siete referencias hace alusión a los términos biological, evolution y social, sus artículos hacen referencia a la teoría de la evolución aplicada al ámbito de lo biológico, social y organizacional; Se analiza la nueva clasificación de los diversos términos utilizados en el contexto de la evolución de los sistemas de fabricación y sus definiciones, inspiradas en la evolución que se manifiesta en la naturaleza. Adicionalmente, se presenta una innovadora relación entre la evolución de los productos manufacturados y la evolución biológica proponiendo el término de cladística, que se utiliza para realizar un seguimiento a las relaciones evolutivas entre los organismos a través de semejanzas compartidas, inspirado en la teoría de la selección natural (EIMaraghy, AlGeddawy \&Azab, 2008).
A partir de la sociobiología y la psicología se analiza el comportamiento a través de la evolución biológica, para ayudar a explicar las conductas sociales, las cuales tienen un potencial significativo para el avance de las ciencias de la organización (Decker \& White, 1999).

Por último, el cluster 3 hace referencia a process, value, management con seis referencias, los escritos de este grupo se centran en la temática de la administración de producción y operaciones, haciendo énfasis en los procesos y teorías en las que se han hecho aplicaciones de conceptos biológicos.

Por ejemplo, la investigación realizada por Chokshi \& McFarlane, (2008) sobre el modelo y enfoque basado en los conceptos de manufactura holónica y gestión de la cadena de suministro, para atender la premisa de que las nuevas presiones de la empresa de personalización de productos industriales y la globalización, llevarán a una mayor necesidad de reconIlustraciónbilidad en plantas de proceso.

\section{Atributos de la vida}

Para el análisis de este eje temático se tuvieron en cuenta cinco palabras, de las cuales se obtuvieron los registros que se visualizan a continuación en la Tabla 1.

Tabla 1. Registros encontrados relacionados con atributos de la vida ${ }^{9}$

\begin{tabular}{|c|c|c|}
\hline Eje & Tema & $\begin{array}{c}\text { Cantidad } \\
\text { de registros }\end{array}$ \\
\hline \multirow{5}{*}{ 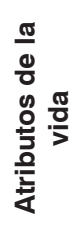 } & ORGANIZACIÓN ORGANICA & 1 \\
\hline & ADAPTATIVO & 26 \\
\hline & EVOLUTIVO & 24 \\
\hline & ORGÁNICO & 1 \\
\hline & ORGANIZACIÓN VIVIENTE & 0 \\
\hline \multicolumn{2}{|c|}{ Registros encontrados } & 52 \\
\hline \multicolumn{2}{|c|}{ Depurados } & $1^{10}$ \\
\hline \multicolumn{2}{|c|}{ Total registros } & 51 \\
\hline
\end{tabular}

9 Elaboración propia.

10 Este documento se depuró ya que al realizar la sinonimización (proceso en el cual se revisa que no existan registros repetidos, sea por autor o título del artículo) a través del programa Reference Manager se encontraba repetido en la base de datos. Fuente de la tabla: elaboración propia. 
Los términos con mayor número de registros encontrados se centraron en los temas adaptativo y evolutivo. En el primer caso, haciendo referencia a la adaptación de la organización al entorno cada vez más competitivo y globalizado, y la rápida respuesta ante los diversos cambios presentados. En el segundo caso, a la evolución de las capacidades organizacionales como una nueva forma de modificar la estructura de manera natural y adecuada a los cambios que propone el entorno.

Adicionalmente, se inscribió a la gestión del nuevo milenio como una ciencia evolutiva. A continuación en la Ilustración 3 se resaltó un crecimiento en el número de escritos que llega a su punto más alto en el año 2006 con 11 publicaciones, en el 2007 tuvo su menor número de publicaciones y volvió y repunto con 10 artículos hasta noviembre de 2008, presentando un comportamiento de leve crecimiento de año a año, reflejando una tendencia de evolución y aumento en la temática relacionada con los atributos de la vida. Es importante anotar que en el año 2000 no se hizo ninguna publicación, al igual que el anterior eje temático.
Se identificaron tres autores con un número de dos publicaciones, sobre un total de 88 autores con un solo escrito. Sus artículos fueron escritos entre los años 2003 y 2007 . Ellos se enfocaron en la teoría evolucionista y los sistemas de adaptación tecnológicos y organizacionales. Armitage autor canadiense, trabajó en el 2007 junto con Plummer en la revista Ecological Economics, en un sistema adaptable complejo, a través de la gestión colaborativa para facilitar el aprendizaje sistemático de la experiencia, en el aprovechamiento y la gestión de los recursos. En el 2005 en la revista Society \& Natural Resources, realizó su artículo de manera individual, en el cual se promueven formas sostenibles de comunidades basadas en la gestión de los recursos y como ellas responden ante los cambios y la incertidumbre, de manera que se genere aprendizaje y capacidades para la adaptación de la gestión. De Inglaterra participa McCarthy, de la Universidad de Sheffield en el Reino Unido, con Technology management - a complex adaptive systems approachen (2003) publicado en el International Journal of Technology Management, haciendo referencia a los métodos de los sistemas y los procesos evolutivos que pueden

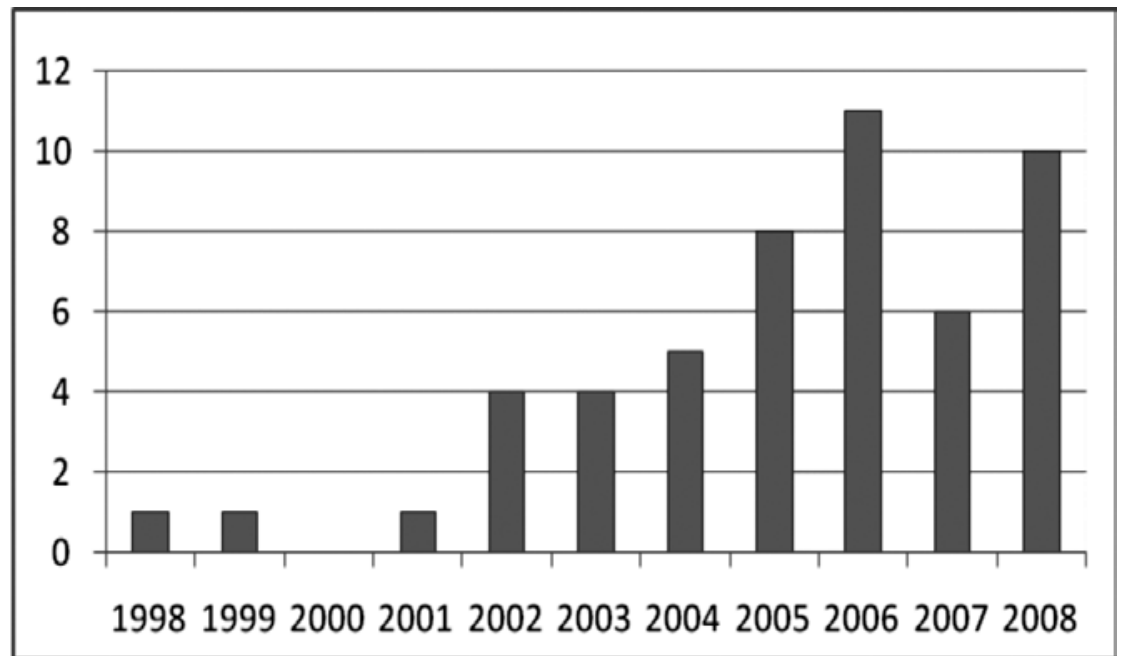

Ilustración 3. Número de publicaciones entre 1998-200811

11 Elaboración propia. 
ayudar a las organizaciones a entender los modelos innovadores y los mecanismos competitivos que influyen en la creación, dirección y explotación de tecnología.

La perspectiva de los sistemas adaptables complejos presentada, ve a las organizaciones como sistemas evolucionando que formulan estrategias por clasificación, selección, adopción y explotación de varias combinaciones de capacidades tecnológicas. En el 2006, McCarthy publicó junto con Tsinopoulos, Allen y Rose-Anderssen, en el Journal of Product Innovation Management, un artículo en el cual se examino el desarrollo de nuevos productos como un sistema adaptable complejo de decisiones, proporcionando un marco conceptual para entender el proceso de innovación dentro de las organizaciones. Winter participó con Murmann, Aldrich y Levinthal en el 2003, y junto con Augier en el 2005. Los dos artículos publicados en el Journal of Management Inquiry, en donde se analiza la aplicación de modelos evolutivos en la gestión y las diferentes teorías administrativas.

Para realizar el análisis de las palabras claves se seleccionaron aquellas que presentaban una frecuencia de aparición igual o mayor a dos. Sin embargo, existe un amplio número de palabras que reflejaron su interés por los temas de gestión con énfasis en los conceptos evolutivos y adaptativos, en los que se incluyeron además, el conocimiento y la innovación como factores claves para la sostenibilidad de una ventaja competitiva. Estas palabras reflejaron la evolución como herramienta hacia las nuevas direcciones y desafíos para crear los nuevos paradigmas dominantes en dirección estratégica, alrededor de los conceptos de conocimiento, aprendizaje, e innovación.

Adicionalmente, los artículos hacían referencia al desarrollo sostenible, el cual requiere cambios en los sistemas socio-técnicos y el cambio social - en las creencias, valores y gobernación que co-evolucionan con los cambios de tecnología, presentado así modelos prácticos para gestionar los procesos de co-evolución: la transición de la gestión y la tecnología. La transición de la gestión, es un modelo de multinivel de gobernación que forma procesos de co-evolución que usa las visiones, las transiciones experimentadas y los ciclos de aprendizaje y adaptación, que hacen parte de estos sistemas complejos. Todos los términos se relacionan con la teoría de la evolución empleados generalmente para el análisis del cambio tecnológico, entre ellos, adaptación, equilibrio, selección, la estrategia, la teoría de la complejidad y el diseño de modelos aplicados a las organizaciones.

De la clasificación se generaron 6 grupos, de los cuales 3 concentraron la mayor cantidad de referencias. Así, el cluster 1 con catorce referencias resaltó los términos relacionados con evolution, process y development. Los artículos que lo componen en su mayoría se centraron en la Teoría de la evolución, como un proceso organizacional de adaptación e innovación, a través de la generación de conocimiento, mejorando los productos y procesos, $y$ por ende la competitividad; por ejemplo, Cattani (2006), analiza el desarrollo de una tecnología sobre la biología evolutiva. Se basa en el concepto de la especialización tecnológica, paralelos a la especiación ecológica, y explica que la especiación se refiere a la aparición de una nueva especie a través de aislamiento reproductivo, en este caso la aparición de una nueva tecnología. Se analizó la reingeniería de procesos, a través del caso de Business Process $R e$-ingeniería y sus familiares-la innovación de procesos y enfoque de la Escuela de Delft-, desde una perspectiva evolutiva sobre la base de una analogía entre los organismos y organizaciones. La investigación presenta un modelo de referencia que se origina en la evolución biológica de las teorías y modelos, para identificar y describir estos procesos evolutivos (Dekkers, 2008).

El cluster 3 con doce referencias hizo alusión a los términos change, value y methodology; El cambio se hace presente en la mayoría de los artículos de este grupo, como un atributo natural no solo del medio ambiente, sino de las organizaciones, la sociedad y los procesos, como una forma de generar nuevas estructuras que permitan ajustarse al entorno y a las nuevas exigencias del mercado, generando valor agregado en sus productos. 
Engdahl, (2005) ilustró un modelo cambio natural circular, centrado en los clientes, rodeado y vinculado a la finalidad, los principios, las personas, la gestión de la filosofía, estructura y funcionamiento, con beneficios valorables como una medida de efecto del logro. Identifica las características principales de los cambios en las estructuras de organización plana, del equipo basado en operaciones y pruebas, afirmando que el éxito de desarrollo de la organización depende de la conciencia de comunicación, la celebración de un valor intrínseco o de creencias, convirtiéndose en un sistema vivo y de colaboración organizacional.

Por último, el cluster 5 hizo referencia a los términos adaptative, resource y natural, con nueve referencias. Sus artículos se centraron en los sistemas complejos adaptativos involucrados en su mayoría con la gestión del conocimiento y la adecuación de procesos, como fuente sostenible de ventajas competitivas.

Adicionalmente se desarrollaron trabajos acerca de la evolución de los sistemas complejos en la aplicación de la gestión de recursos naturales, en los cuales la sostenibilidad no puede ser un objetivo estático, debe ser un proceso evolutivo de mejora de la gestión de los sistemas socio-ecológicos, a través de una mejor comprensión y conocimiento.

\section{Conceptos de integración}

En la Tabla 2 se puede observar el volumen de datos bibliográficos obtenidos. Se observó que la mayor cantidad de registros corresponden al tema ecológico, como una forma de organización y estructuración; las redes, como sistema de colaboración y recursos compartidos para la mejora de la productividad y eficiencia en las organizaciones, igualmente relacionado con los sistemas de información. La inercia y la mortalidad también hacen parte de los temas con mayor número de registros. Al revisar la Ilustración 4, la cual muestra la evolución de las publicaciones en la temática propuesta, se observo una tendencia creciente de 1998 al 2006, donde alcanzó su punto máximo con 22 publicaciones. Sin embargo, en los años subsiguientes existió una tendencia decreciente de publicaciones referente al tema.

Tabla 2. Registros encontrados relacionados con conceptos de integración ${ }^{12}$

\begin{tabular}{|c|c|c|}
\hline Eje & Tema & $\begin{array}{l}\text { Cantidad } \\
\text { de registros }\end{array}$ \\
\hline \multirow{12}{*}{ 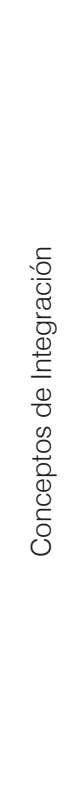 } & SOCIOBIOLOGÍA & 1 \\
\hline & ECOSISTEMA & 5 \\
\hline & ECOLOGÍA & 28 \\
\hline & MORTALIDAD & 13 \\
\hline & $\mathrm{NICHO}$ & 9 \\
\hline & SINERGÍA & 3 \\
\hline & INERCIA & 20 \\
\hline & COOPERACIÓN & 7 \\
\hline & CLUSTER & 12 \\
\hline & CONGLOMERADO & 5 \\
\hline & AGLOMERACIÓN & 6 \\
\hline & RED & 22 \\
\hline \multicolumn{2}{|c|}{ Registros encontrados } & 131 \\
\hline \multicolumn{2}{|c|}{ Depurados } & 7 \\
\hline \multicolumn{2}{|c|}{ Total registros } & 124 \\
\hline
\end{tabular}

12 Elaboración propia. 
En relación con los autores con mayor número de publicaciones se encontró una tendencia más clara que en los anteriores ejes temáticos, señalando que de los 210 autores que han escrito sobre el tema, se destacaron seis con un número igual o mayor a 3 artículos. En la Ilustración 5 se muestran los resultados.

El autor más destacado fue M.T. Hannan con 11 publicaciones, mostrando una continuidad desde el 2001 al 2006, con un promedio de dos artículos por año. El tema de sus publicaciones está enfocado hacía la organización ecológica, vista desde diversas características, como la inercia, el nicho, la mortalidad, como una forma de organización y estructuración frente al cambio organizacional, retomando la teoría sociológica aplicada a las organizaciones. Igualmente este autor ha trabajado en conjunto con G.R. Carroll, L. Polos, J.H. Freeman y J.N. Baron quienes también hacen parte de los principales autores en este eje temático.

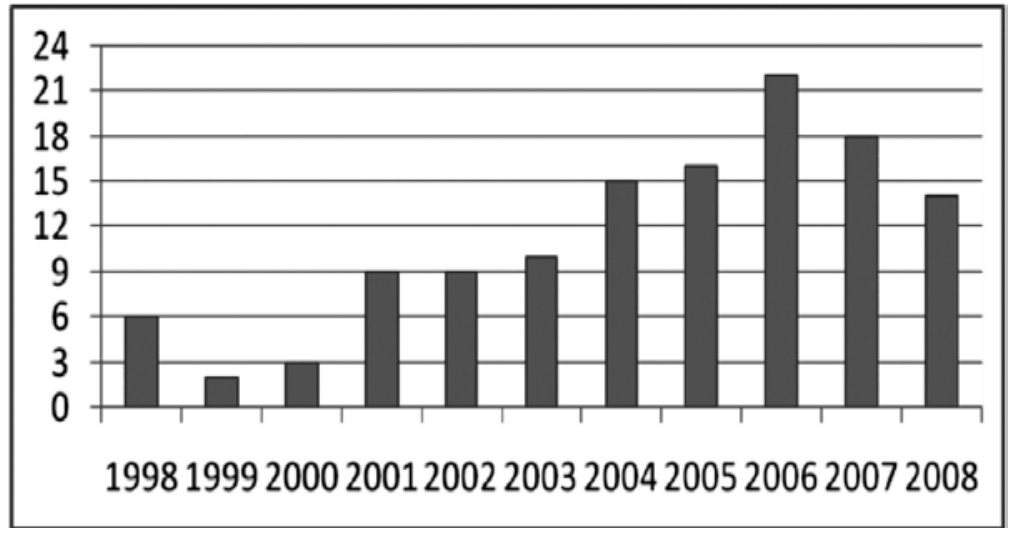

Ilustración 4. Número de publicaciones entre los años 1998-2008 ${ }^{13}$

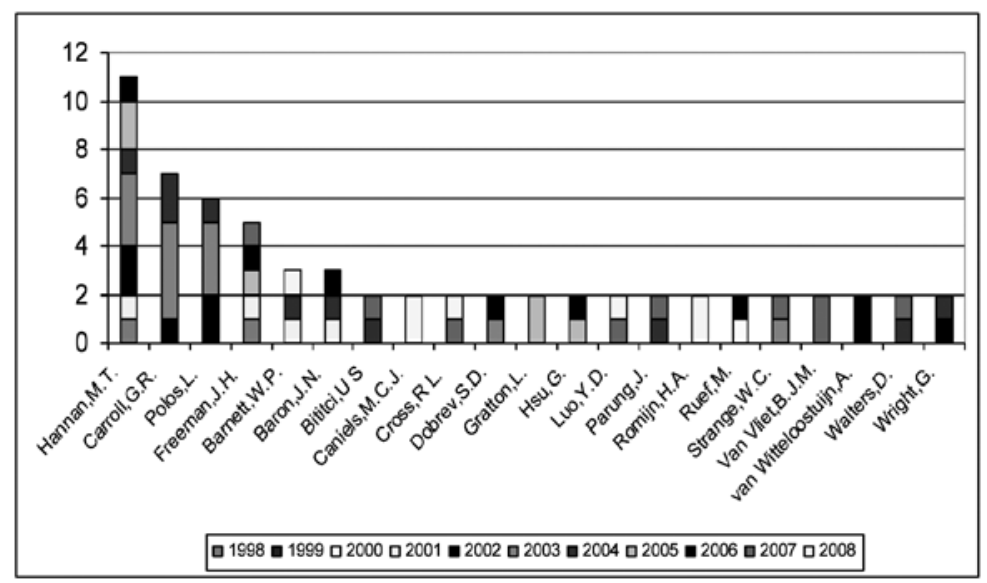

Ilustración 5. Autores con mayor número de publicaciones ${ }^{14}$

13 Elaboración propia.

14 Elaboración propia. 
En los artículos "Cascading organizational change" $y$ "The fog of change: Opacity and asperity in organizations" escritos en 2003 por Hannan, Polos y Carrol se desarrolla una teoría formal de los aspectos estructurales de cambio orgánico. Se concentran en los cambios de la arquitectura de una organización, diagramada como un sistema del código. Modelan el proceso común de cómo un cambio arquitectónico inicial incita otros cambios en la organización, mientras va generando una cascada de cambios que representan la reorganización completa. Igualmente en el 2003 publicaron el artículo "The organizational niche" en el cual adelantan una reconstrucción teórica del concepto de nicho, con aplicación especial en las organizaciones. El modelo formal propuesto se unifica en varias líneas activas de la teoría sociológica. También extiende la noción del nicho del dominio de conductas aplicadas a las reglas que codifican identidades sociales y las formas organizacionales.

La reconstrucción da la visión más profunda en el nicho de una población organizacional así como las organizaciones individuales. Los demás autores hacen énfasis en las formas de integración, aglomeración y clusters, como fuente de competitividad, adaptabilidad y sostenibilidad en el entorno. Las revistas con mayor número de publicaciones, fueron Organization Science, Industrial and Corporate change, California Management Review y Organization Studies, quienes tienen respectivamente 7 , 6, 5 y 4 publicaciones. El criterio de selección fue igual o mayor a dos publicaciones, de un total de 79 revistas se seleccionó 23 de ellas.

$\mathrm{Al}$ analizar las palabras claves que se obtuvieron con una frecuencia de aparición igual o mayor a diez veces, se encontraron conceptos tales como ecología, evolución, competencia, inercia, innovación, conocimiento, nicho, sistemas complejos o teoría de la complejidad, clusters, aglomeración, mortalidad, etc., que hacían referencia a sistemas de integración con la aplicación de conceptos biológicos.

Se creó un mapa tecnológico de este eje temático, representado por cuatro grupos que aglutinaron la mayoría de referencias. El cluster 5 contó con el $33 \%$ del total de artículos con 41 referencias, las cuales resaltaron los términos organizational model, change, ecology, inertia y population; la temática está centrada en la ecología organizacional, que es la teoría que estudia las poblaciones de organizaciones, tomando a la organización individual como la unidad de observación. Debido a la inercia (incapacidad de las organizaciones de cambiar tan rápidamente como el ambiente), se ve como un atributo prominente de las organizaciones el que ocurran cambios en su población, mayormente a través de un proceso de nacimiento y muerte organizacional en contraste con la adaptación y la supervivencia de organizaciones individuales. La ecología organizacional investiga los antecedentes, el desempeño y las consecuencias de un proceso de selección ambiental (Boyer \& Robert, 2006; Singh, 2006).

Becker, (2007) en su artículo introdujo el concepto de ecología organizacional como marco de referencia para la reflexión sobre el lugar de trabajo, al describirlo como una compleja red de interdependencia social y factores organizacionales que en combinación, influyen en la comunicación informal, la interacción y los patrones de aprendizaje. En concreto, propuso los conceptos de "dinámica limitada" y "dinámica armónica" para explicar por qué las intervenciones organizacionales implican la manipulación social o de factores físicos que a menudo no logran alcanzar los objetivos propuestos. También examinó cómo los aspectos espaciales de la ecología de una organización pueden contribuir a una comunicación más eficaz y a mejores patrones de interacción que el apoyen el aprendizaje informal.

El cluster 7 con dieciséis referencias, es decir el $12.9 \%$ del total de escritos, hizo alusión a los términos network, structure, value, integration y development. Entre los artículos más destacados de este grupo, se encontraron el de Zeleny (2001), en el cual examinó la naturaleza de las redes de pequeñas y medianas empresas, tales como los distritos industriales en Italia y en los grupos en los EE.UU. de Silicon Valley. A su vez, afirma que muchos de los estudios de estas redes no han logrado entender su naturaleza, con el argumento de que estas redes se caracterizan por su organización circular de la 
formación de procesos productivos, que son autopoiéticos, es decir, se auto- producen, de forma que ellos son cerrados organizacionalmente y estructuralmente son sistemas abiertos. Adicionalmente, sustentó que estas características son fundamentales para la sostenibilidad de las redes.

El cluster 1 con el 11.29\% de participación se centro en las palabras organizational, network, structure, change y process con 14 referencias. Los artículos hacían referencia a la forma en que la estructura informal y la cultura de la organización pueden producir resistencia a las iniciativas de cambio. Sugiere que la resistencia al cambio puede ser mejor comprendida, al reunir los marcos estructurales y culturales, que combinan una conciencia de que las personas o grupos se sientan en una red con un mayor reconocimiento y práctica de sus valores culturales.

Por último, el cluster 4 tiene 9 referencias y cuenta con los siguientes términos, Management, development, resource, learn, ecological y structure. Se describen las políticas de asistencia de alta tecnología para las pequeñas empresas, que a lo largo de los últimos años se han dirigido a fomentar su investigación y desarrollo $(\mathrm{I}+\mathrm{D})$ a través de redes de colaboración y transferencia de tecnología. Se retomó nuevamente el tema de ecología organizacional y la adecuación de estructuras a través de las teorías de cambio y adaptación.

\section{Discusión}

Esta primera aproximación permite a los investigadores en el área de teoría organizacional visualizar las oportunidades que tiene la aplicación de la metáfora biológica a los conceptos empresariales. Si bien es cierto, esta es una tendencia reciente, puede vislumbrarse a través del análisis bibliométrico que ha venido aumentando en importancia. Los conceptos de ciclo de vida, algoritmos genéticos, adaptativo, evolutivo, coevolución, ecología, inercia, clúster y natalidad y mortalidad, no sólo son utilizados exitosamente en las ciencias administrativas y económicas (Montoya, 2010), sino también en otras áreas de las ciencias sociales. Estos han permitido entender la realidad y modelarla para entenderla y así poder impactarla positivamente.

Esta revisión puede resultar de mucha utilidad en las comunidades de investigación para enfocar la aplicación de las metáforas biológicas en las nuevas formas de organizaciones, en este sentido es posible crear nuevos conceptos basados en conceptos biológicos, explicar fenómenos empresariales con mayor integralidad usando la metáfora y lograr mayor entendimiento de los fenómenos organizacionales con estas aplicaciones. También resulta particularmente útil en los modelos pedagógicos para explicar aproximaciones nuevas de las teorías organizacionales.

El estudio realizado permite observar además un proceso de legitimización de la metáfora desde los años setentas cuando surge la sociobiología como ciencia con Wilson (2002) y los aportes de Hannan \& Freeman (1977). En Latinoamérica se ha venido trabajando estos conceptos, en particular en Colombia se tienen aportes con los trabajos de Illera (1982), Castellanos et al. (2002), Montoya et al. (2009, 2010, 2011), Ochoa \& Montoya (2010), entre otros más.

Los investigadores en gestión pueden adoptar estos enfoques que resultarán sin duda creativos y permitentes para entender una problemática que comparte con el sistema complejo biológico. Las organizaciones en el actual entorno dependen de nuevos papeles, actitudes, habilidades y procedimientos que deben ser aprendidos a ciertos costos, en ocasiones estos papeles deben ser inventados, lo cual lleva a importantes restricciones de todos los niveles, lo cual puede solucionarse gracias a diferentes relaciones empresariales (Morales, 2002).

La gestión de organizaciones y su estudio a través de la teoría organizacional y la aplicación de la metáfora biológica busca alternativas para permitir un mejor desempeño de las mismas y una mejor adaptación a los cambios del entorno, de la facilidad de su adaptación depende, en gran medida que tenga la capacidad de supervivir y crecer, como objetivos básicos organizacionales (Serrano \& Villareal, 1997), objetivos que comparte con los ecosistemas y los seres vivos. 
La provocación consiste en encontrar metáforas que sean realmente de máximo valor (Cadavid, 2010) en las cuales puedan encontrarse los modelos biológicos y organizacionales para proporcionar oportunidades de entendimiento y desarrollo.

\section{Conclusiones}

La metáfora se presenta como una alternativa de desarrollo conceptual que posibilita hacer construcciones a partir de conocimientos previos de otras ciencias y que permite entender en elementos de otros conceptos, el desarrollo de las empresas y su gestión. Es así como palabras y contextos que pueden ser aplicados en otras ciencias resultan pertinentes en la teoría organizacional, tal como se describió a partir de conceptos pertenecientes a las ciencias biológicas.

El análisis realizado muestra que la dinámica actual de publicaciones sobre el aporte de las biociencias a la gestión ha sido limitado, adicionalmente es muy diverso conceptualmente, no existen aún corrientes o temáticas claramente definidas.

La literatura más desarrollada o concentrada del tema se identifica en el eje temático de conceptos de integración, con un énfasis hacia la formación de comunidades ecológicas y nichos, donde se evalúan las estructuras organizacionales con un enfoque hacía la organización ecológica, vista desde diversas características, como la inercia, el nicho, la mortalidad, como una forma de organización y estructuración frente al cambio organizacional, retomando la teoría sociológica aplicada a las organizaciones.

Un elemento fundamental dentro de los conceptos de integración empresarial basados en metáforas biológicas tiene que ver con los procesos coevolutivos, las organizaciones vistas como seres biológicos también tienen que realizar un importante esfuerzo para adaptarse, crecer y coexistir en un medio ambiente que es altamente competitivo. Dichas metáforas permiten, a través de realizar sinergias, que las organizaciones se adapten entre sí y de esta manera puedan desarrollar ventajas adaptativas en el mercado.

No existe una tendencia claramente definida que indique hacía donde se dirige la temática general, no se muestra ni creciente ni decreciente, lo que permite prever que la temática estudiada es realmente nueva y proporciona interesantes posibilidades a los investigadores en gestión empresarial. Por el contrario, existe un fuerte posicionamiento de la aplicación de los conceptos biológicos en la implementación de procesos productivos, como una forma de administrar eficientemente la producción.

Adicionalmente, se generan metáforas fuertemente relacionadas con las formas de organización del medio ambiente para ser aplicadas a las estructuras organizacionales. Los aportes encontrados a través de la vigilancia desarrollada hacen poco énfasis a desarrollos de carácter conceptual, trasladándose a la aplicación de conceptos biológicos para desarrollar herramientas y técnicas que permiten una mejor operacionalización en el campo de la gestión, en este campo también existe un gran potencial.

\section{Referencias}

Armitage D.R. (2005). Community-based Narwhal management in Nunavut, Canada: Change, uncertainty, and adaptation. En: Society \& Natural Resources, 18 (8): 715-731.

Armitage, D.R. \& Plummer, R. (2007). A resilience-based framework for evaluating adaptive co-management: Linking ecology, economics and society in a complex world. En: Ecological Economics, 61 (1): 62-74.

Becker, F. (2007). Organizational ecology and knowledge networks. En: California Management Review, 49 (2): 42.

Beer, S. (1977) Cibernética y Administración. Stanfford. Sexta Impresión, México.

Bertalanffy, L. (1976). Teoría General de los Sistemas. Fondo de Cultura Económica, México.

Boyer, M. \& Robert, J. (2006). Organizational inertia and dynamic incentives. En: Journal of Economic Behavior \& Organization, 59 (3): 324-348.

Burnes, B. (2005). Complexity theories and organizational change. En: International Journal of Management Reviews, 7 (2): 73-90.

Cadavid, L. (2010). Propuesta para la medición del ajuste entre las metáforas organizacionales y las herramientas de modelado. Facultad de Minas, Universidad Nacional de Colombia, Medellín. 
Castellanos, O., \& Montoya, A. (2002). Biogestión. En: Revista Innovar, 18: 55.

Castellanos, O., Montoya, A., \& Montoya, I. (2004). La Gerencia Genética: una metáfora biológica aplicada a la gestión de la Biotecnología. En: Revista Innovar, 24: 93-104.

Cattani, G. (2006). Technological pre-adaptation, speciation, and emergence of new technologies: how corning invented and developed fibre optics. En: Industrial \& Corporate Change, 15 (2): 285-318.

Chokshi, N. \& McFarlane, D. (2008). A distributed architecture for reconllustraciónble control of continuous process operations. En: Journal of Intelligent Manufacturing, 19 (2): 215232.

Decker, P. \& White, R. (1999). The Evolution of Social Structure: Why Biology Matters. En: The Academy of Management Review, 24 (4): 843-853.

Dekkers, R. (2008). Adapting organizations: The instance of business process re-engineering. En: Systems Research and Behavioral Science, 25 (1): 45-66.

ElMaraghy, H., AlGeddawy, T. \& Azab, A. (2008). Modelling evolution in manufacturing: A biological analogy. En: CIRP Annals-Manufacturing Technology, 57 (1): 467-472.

Engdahl, R.A. (2005). Organization evolution: the natural change model for organizational structure in changing times. En: Organization Development Journal,23 (2): 50-60.

Gell - Mann, M. (1994). El Quark y El Jaguar. Aventuras en lo simple y en lo complejo. Tusquets Editores. Barcelona.

Hannan, T., \& Freeman, J. (1977). The Population Ecology of Organizations. En: The American Journal of Sociology, Vol. 82, No. 5. (Mar,): 929-964.

Hannan, M.T., Polos, L., \& Carroll, G.R. (2003). Cascading organizational change. En: Organization Science, 14 (5): 463482.

Hannan, M.T., Polos, L., \& Carroll, G.R. (2003). The fog of change: Opacity and asperity in organizations. En: Administrative Science Quarterly, 48 (3): 399-432.

Hannan, M.T., Polos, L., \& Carroll, G.R. (2003). The organizational niche. En: Sociological Theory, 21 (4): 309-340.

Illera, E. (1982). Anatomía y Fisiología de la Organización. Universidad de la Salle, Bogotá.

Juttner, U., Godsell, J., \& Christopher, M.G. (2006). Demand chain alignment competence - delivering value through product life cycle management. En: Industrial Marketing Management, 35 (8): 989-1001.

Koontz, H, (2008). Administración una perspectiva global. McGraw Hill, Bogotá.

Liao, J. \& Stonebraker, P.W. (2006). Supply chain integration: exploring product and environmental contingencies. En: Supply Chain Management: An International Journal, 11 (1): 34-43.

Lores, J. (2006). Metáforas, Estilos Y Paradigmas. Universidad de Lleida, España.

McCarthy, I.P. (2003). Technology management - a complex adaptive systems approach. En: International Journal of Technology Management, 25 (8): 728-745.

McCarthy, I.P., Tsinopoulos, C., Allen, P., \& Rose-Anderssen, C. (2006). New product development as a complex adaptive system of decisions. En: The Journal of Product Innovation Management, 23 (5): 437-456.
Mendoza, V. (2005). Metáfora: Racionalidad Comunicativa y Responsabilidad Ética. En: Revista Número Actual. Universidad del Valle, México, 35.

Montoya, A. (1999). Gerencia Genética. Tesis de Maestría en Administración, Bogotá, Universidad Nacional de Colombia.

Montoya, A. (2010). Gestión de Sistemas de Integración empresarial desde una perspectiva Biológica. Tesis de programa Doctoral en Ciencias Económicas, Bogotá, Universidad Nacional de Colombia.

Montoya, A., Montoya, I., \& Castellanos, O. (2010). Ponencia: Características de una aproximación biológica para el estudio de mecanismos de integración empresarial. Congreso Internacional de Investigación en Ciencias Administrativas. El Rol de las Ciencias Administrativas en el Desarrollo Social. Tecnológico de Monterrey, Monterrey (Abril de 2010).

Montoya, A., Robayo, P., Castellanos, O., \& Fonseca, S. (2008). Fortalecimiento conceptual del Grupo de Investigación y Desarrollo, en gestión, productividad y competitividad: Identificación de tendencias sobre el aporte de las biociencias a la Gestión. Proyecto de Investigación no publicado, Bogotá, Universidad Nacional de Colombia.

Montoya, A. (2010). Gestión de sistemas de integración empresarial bajo la perspectiva biológica. Tesis de Doctorado, Universidad Nacional de Colombia, Bogotá.

Montoya, A., Montoya I. \& Castellanos, O. (2009 (a)). Modelos de ecosistemas como alternativa para las soluciones medio ambientales, estudio de caso. En: XIII Congreso Internacional de Investigación en Ciencias Administrativas, ACACIA, La administración frente a la globalización: Gobernabilidad y desarrollo, Universidad Autónoma Metropolitana UAM, México D.F. (Julio de 2009).

Montoya, A., Montoya, I. \& Castellanos, O. (2010). Características de una aproximación biológica para el estudio de mecanismos de integración empresarial, En: XIV Congreso Internacional de Investigación en Ciencias Administrativas, El Rol de las Ciencias Administrativas en el Desarrollo Social Tecnológico de Monterrey, Monterrey, (Abril de 2010).

Montoya, A., Montoya, I. \& Rojas, S.(2011). Enfoques Biológicos de la Gestión: desde la competencia hasta la cooperación, XV Congreso Internacional de Investigación en Ciencias Administrativas Boca del Río, Veracruz, 17 al 20 de mayo de 2011.

Montoya, L., Montoya, I. \& Castellanos, O. (2010). La metáfora organizacional: alternativa de entendimiento procedente de otras ciencias. En: Revista de la Facultad de Ciencias Económicas de la Universidad Militar Nueva Granada. rev.fac. cienc.econ, XVIII (1)

Morales, J. (2002). Decaimiento, mortandad y sobrevivencia organizacional: Gestión estratégica de las Organizaciones Mexicanas. En: El neoliberalismo, Denarius, 5, (1): 55-73.

Morgan, G. (1998). Imágenes de la Organización. México, Alfaomega.

Ochoa, D. \& Montoya, A.(2010). Consorcios microbianos: una metáfora biológica aplicada a la asociatividad empresarial en cadenas productivas agropecuarias. En: Revista Facultad de Ciencias Económicas, Universidad Militar Nueva ranada, XVIII (2): 55-74.

Serrano, J. \& Villareal, J. (1997). Fundamentos de Finanzas. Segunda Edición. Mcgraw Hill: México. 
Singh, J.V. (2006). Ecology, strategy and organizational change. En: Ecology and Strategy, 23: 177-214.

Wilson, E.(2002). Sociobiology, The new synthesis. 25Th Anniversary ed. Harvard Collage.

Winter, S.G. \& Augier, M. (2005). Why is management an evolutionary science? An interview with Sidney G. Winter. En: Journal of Management Inquiry, 14 (4): 344-354.
Winter, S., Murmann, J., Aldrich, H. \& Levinthal, D. (2003). Evolutionary thought in management and organization theory at the beginning of the new millennium - A symposium on the state of the art and opportunities for future research. EN: Journal of Management Inquiry, 12 (1): 22-40.

Zeleny, M. (2001). Autopoiesis (self-production) in SME networks. EN: Human Systems Management, 20(3): 201-207. 